\title{
Ptolemy diagrams and torsion pairs in the cluster category of Dynkin type $A_{n}$
}

\author{
Thorsten Holm • Peter Jørgensen • Martin Rubey
}

Received: 19 October 2010 / Accepted: 9 March 2011 / Published online: 31 March 2011

(C) Springer Science+Business Media, LLC 2011

\begin{abstract}
We give a complete classification of torsion pairs in the cluster category of Dynkin type $A_{n}$. Along the way we give a new combinatorial description of Ptolemy diagrams, an infinite version of which was introduced by $\mathrm{Ng}(1005.4364 \mathrm{v} 1$ [math.RT], 2010). This allows us to count the number of torsion pairs in the cluster category of type $A_{n}$. We also count torsion pairs up to Auslander-Reiten translation.
\end{abstract}

Keywords Clique $\cdot$ Cluster algebra Cluster tilting object $\cdot$ Generating function . Recursively defined set $\cdot$ Species $\cdot$ Triangulated category

This work has been carried out in the framework of the research priority programme SPP 1388 Darstellungstheorie of the Deutsche Forschungsgemeinschaft (DFG). We gratefully acknowledge financial support through the grant HO 1880/4-1.

T. Holm · M. Rubey

Institut für Algebra, Zahlentheorie und Diskrete Mathematik, Fakultät für Mathematik und Physik, Leibniz Universität Hannover, Welfengarten 1, 30167 Hannover, Germany

T. Holm

e-mail: holm@math.uni-hannover.de

url: http://www.iazd.uni-hannover.de/ tholm

M. Rubey

e-mail: martin.rubey@ math.uni-hannover.de

url: http://www.iazd.uni-hannover.de/ rubey

P. Jørgensen ( $\varangle)$

School of Mathematics and Statistics, Newcastle University, Newcastle upon Tyne NE1 7RU, UK e-mail: peter.jorgensen@ncl.ac.uk url: http://www.staff.ncl.ac.uk/peter.jorgensen 


\section{Introduction}

Let $\mathscr{A}$ be the cluster algebra of Dynkin type $A_{n}$, let $\mathrm{C}$ be the cluster category of Dynkin type $A_{n}$, and let $P$ be a (regular) $(n+3)$-gon. There are bijections between the following sets:

(i) Clusters in $\mathscr{A}$,

(ii) Cluster tilting objects in $\mathrm{C}$,

(iii) Triangulations by non-crossing diagonals of $P$.

See Caldero, Chapoton, and Schiffler [7] and Iyama [10].

To place this in a larger context, note that if $u$ is a cluster tilting object in $\mathrm{C}$ and $\mathrm{U}=\operatorname{add}(u)$ is the full subcategory consisting of direct sums of direct summands of $u$, then $(U, \Sigma U)$ is a so-called torsion pair by Keller and Reiten [13, Sect. 2.1]. Here $\Sigma$ is the suspension functor of the triangulated category $C$. The triangulation on $C$ is due to Keller [12] and is based on the definition of $C$ as an orbit category by Buan, Marsh, Reineke, Reiten, and Todorov [6].

In this paper, we widen the perspective by investigating general torsion pairs in $C$. A torsion pair in a triangulated category $\mathrm{T}$ is a pair $(\mathrm{X}, \mathrm{Y})$ of full subcategories closed under direct sums and direct summands such that

(i) The morphism space $\mathrm{T}(x, y)$ is zero for $x \in \mathrm{X}, y \in \mathrm{Y}$;

(ii) Each $t \in \mathrm{T}$ sits in a distinguished triangle $x \rightarrow t \rightarrow y \rightarrow \Sigma x$ with $x \in \mathrm{X}, y \in \mathrm{Y}$.

This concept was introduced by Iyama and Yoshino in [11, Definition 2.2]. It is a triangulated version of the classical notion of a torsion pair in an abelian category due to Dickson, see [8]. In the triangulated situation, it has precursors in the form of the t-structures of Beilinson, Bernstein, and Deligne, where, additionally, one assumes $\Sigma \mathrm{X} \subseteq \mathrm{X}$ (see [2]), and the co-t-structures of Bondarko and Pauksztello where, additionally, one assumes $\Sigma^{-1} \mathrm{X} \subseteq \mathrm{X}$ (see [5, 16]). Note that the terminology of torsion pairs in triangulated categories was also employed by Beligiannis and Reiten in [3], but they used it as a synonym for $\mathrm{t}$-structures.

There has so far been little systematic investigation of torsion pairs in triangulated categories, but $\mathrm{Ng}$ [15] gave a complete classification of torsion pairs in the cluster category of type $A_{\infty}$ in terms of certain infinite combinatorial objects. See [9] for details on this category. In particular, Ng introduced the Ptolemy condition which, when supplanted to the finite situation, takes the following form: a Ptolemy diagram is a set of diagonals of a finite polygon (with a distinguished oriented base edge) such that, if the set contains crossing diagonals $\mathfrak{a}$ and $\mathfrak{b}$, then it contains all diagonals which connect end points of $\mathfrak{a}$ and $\mathfrak{b}$. See Fig. 1 and Definition 2.1 below.

For instance, a polygon with no diagonals (an "empty cell") is a Ptolemy diagram, as is a polygon with all diagonals (a "clique"). The triangle is the only Ptolemy diagram which is both an empty cell and a clique. If $A$ and $B$ are boundary edges of two Ptolemy diagrams, then there is an obvious way of gluing $A$ to $B$ to obtain a new Ptolemy diagram. We will show the following classification result on Ptolemy diagrams and torsion pairs. 
Fig. 1 The Ptolemy condition

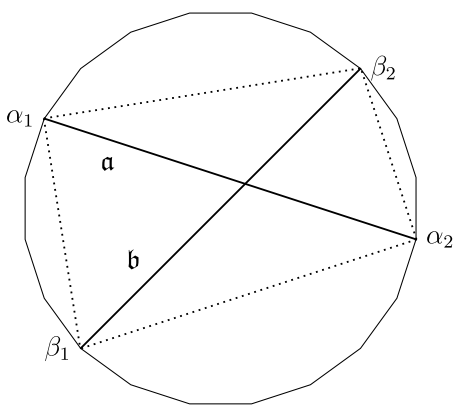

\section{Theorem A}

(i) There is a bijection between Ptolemy diagrams of the $(n+3)$-gon and torsion pairs in the cluster category $\mathrm{C}$ of Dynkin type $A_{n}$.

(ii) Each Ptolemy diagram can be obtained by gluing empty cells and cliques.

Note that a triangulation by non-crossing diagonals is a Ptolemy diagram. Under the bijection of part (i), it corresponds to a torsion pair coming from a cluster tilting object.

Part (i) is a type $A_{n}$ analogue of Ng's classification, but our proof is easier than hers because it uses the gluing in part (ii). The gluing follows from the observation that if a diagonal in a Ptolemy diagram crosses no other diagonal in the diagram, then it divides the diagram into two smaller Ptolemy diagrams. In fact, the gluing can be organised so as to be unique, and this permits us to prove the following counting result which, by virtue of part (i), also counts torsion pairs in $\mathrm{C}$.

Theorem B The number of Ptolemy diagrams of the $(n+3)$-gon is

$$
\frac{1}{n+2} \sum_{\ell \geq 0} 2^{\ell}\left(\begin{array}{c}
n+1+\ell \\
\ell
\end{array}\right)\left(\begin{array}{c}
2 n+2 \\
n+1-2 \ell
\end{array}\right)
$$

with the convention that the second binomial coefficient is 0 for $n+1-2 \ell<0$.

The first few values, starting at $n=0$, are

$$
\begin{aligned}
& 1,4,17,82,422,2274,12665,72326,421214,2492112, \\
& 14937210,90508256,553492552,3411758334,21175624713, \\
& 132226234854,830077057878, \ldots
\end{aligned}
$$

This sequence may not have appeared previously in the literature. Based on this paper, it is now item A181517 in the Online Encyclopedia of Integer Sequences [18]. Its asymptotic behaviour can be determined explicitly, see Remark 3.2.

We are also able to determine the generating function for Ptolemy diagrams up to rotation, see Proposition 3.5. This corresponds to counting torsion pairs up to 
Auslander-Reiten translation. The first few values are

$$
\begin{aligned}
& 1,3,5,19,62,301,1413,7304,38294,208052, \\
& 1149018,6466761,36899604,213245389,1245624985, \\
& 7345962126,43688266206, \ldots
\end{aligned}
$$

Again it seems that this sequence was not encountered before. It is now item A181519 in the Online Encyclopedia of Integer Sequences.

Köhler [14] recently classified and counted thick subcategories of triangulated categories with finitely many indecomposables. This is the same as counting torsion pairs $(X, Y)$ in which $X$ and $Y$ are triangulated subcategories; these are known as stable $\mathrm{t}$-structures. One can show that the only stable t-structures in the cluster category $\mathrm{C}$ are $(C, 0)$ and $(0, C)$, so our results do not overlap with Köhler's.

\section{Characterizing torsion pairs combinatorially}

Let $P$ be an $(n+3)$-gon with a distinguished oriented edge which we refer to as the distinguished base edge. We denote vertices of the polygon by lower case Greek letters. An edge is a set of two neighbouring vertices of the polygon. A diagonal is a set of non-neighbouring vertices. Two diagonals $\left\{\alpha_{1}, \alpha_{2}\right\}$ and $\left\{\beta_{1}, \beta_{2}\right\}$ cross if their end points are all distinct and come in the order $\alpha_{1}, \beta_{1}, \alpha_{2}, \beta_{2}$ when moving around the polygon in one direction or the other. This corresponds to an obvious notion of geometrical crossing. Note that a diagonal does not cross itself and that two diagonals sharing an end point do not cross.

We recall the following from the introduction.

Definition 2.1 Let $\mathfrak{A}$ be a set of diagonals in $P$. Then $\mathfrak{A}$ is a Ptolemy diagram if it has the following property: when $\mathfrak{a}=\left\{\alpha_{1}, \alpha_{2}\right\}$ and $\mathfrak{b}=\left\{\beta_{1}, \beta_{2}\right\}$ are crossing diagonals in $\mathfrak{A}$, then those of $\left\{\alpha_{1}, \beta_{1}\right\},\left\{\alpha_{1}, \beta_{2}\right\},\left\{\alpha_{2}, \beta_{1}\right\},\left\{\alpha_{2}, \beta_{2}\right\}$ which are diagonals are in $\mathfrak{A}$. See Fig. 1.

Note that, because of the distinguished base edge which we draw in bold, the two Ptolemy diagrams in Fig. 2 are distinct.

Let $\mathrm{C}$ be the cluster category of type $A_{n}$. There is a bijection between indecomposable objects of $\mathrm{C}$ and diagonals of $P$. We use lower case roman letters for (indecomposable) objects of $\mathrm{C}$ and lower case fraktur letters for the corresponding diagonals.

Fig. 2 Two different Ptolemy diagrams
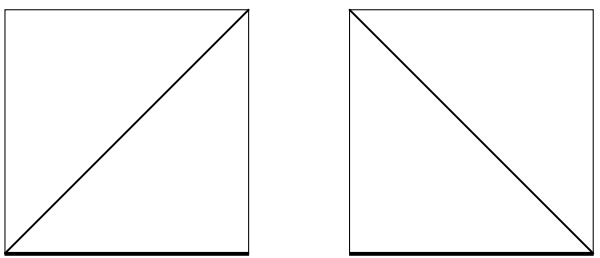
Fig. 3 The dotted diagonals are nc of the solid diagonals

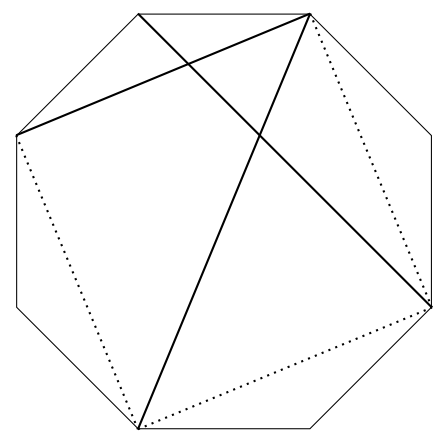

The suspension functor $\Sigma$ acts on (indecomposable) objects and hence on diagonals; the action on diagonals is rotation by one vertex. Note that $\Sigma$ is equal to the Auslander-Reiten translation of $C$ since $C$ is 2-Calabi-Yau. We have

$$
\operatorname{dim} \operatorname{Ext}_{\mathrm{C}}^{1}(a, b)= \begin{cases}1 & \text { if } \mathfrak{a} \text { and } \mathfrak{b} \text { cross }, \\ 0 & \text { otherwise, }\end{cases}
$$

see [7].

The bijection between indecomposable objects of $\mathrm{C}$ and diagonals of $P$ extends to a bijection between subcategories of $C$ closed under direct sums and direct summands, and sets of diagonals of $P$. We use upper case sans serif letters for subcategories and upper case fraktur letters for the corresponding sets of diagonals. The suspension functor acts on diagonals and hence on sets of diagonals.

Definition 2.2 If $\mathfrak{A}$ is a set of diagonals, then

$$
\text { nc } \mathfrak{A}=\{\mathfrak{b} \text { is a diagonal of } P \mid \mathfrak{b} \text { crosses no diagonal in } \mathfrak{A}\} \text {. }
$$

Figure 3 is an example where $\mathfrak{A}$ consists of the solid diagonals and nc $\mathfrak{A}$ of the dotted ones. Note that this is not a Ptolemy diagram. In the example, $\mathfrak{A}$ and nc $\mathfrak{A}$ are disjoint but this is not always the case since a diagonal does not cross itself.

Let $A$ be a subcategory of $C$ closed under direct sums and direct summands. We define the perpendicular subcategories by

$$
\begin{aligned}
& { }^{\perp} \mathrm{A}=\{c \in \mathrm{C} \mid \mathrm{C}(c, a)=0 \text { for each } a \in \mathrm{A}\}, \\
& \mathrm{A}^{\perp}=\{c \in \mathrm{C} \mid \mathrm{C}(a, c)=0 \text { for each } a \in \mathrm{A}\} .
\end{aligned}
$$

If $A$ corresponds to the set of diagonals $\mathfrak{A}$, then (1) implies that ${ }^{\perp} \mathrm{A}$ corresponds to $\Sigma^{-1}$ nc $\mathfrak{A}$ and $\mathrm{A}^{\perp}$ corresponds to $\Sigma \mathrm{nc} \mathfrak{A}$; this follows using $\mathrm{C}(c, \Sigma d)=\operatorname{Ext}_{\mathrm{C}}^{1}(c, d)$. Note that the operator nc commutes with $\Sigma$ and $\Sigma^{-1}$.

Proposition 2.3 The following are equivalent for a subcategory $\mathrm{A}$ of $\mathrm{C}$ which is closed under direct sums and direct summands.

(i) A is closed under extensions, that is, if $a_{1}, a_{2} \in \mathrm{A}$ and $a_{1} \rightarrow b \rightarrow a_{2} \rightarrow \Sigma a_{1}$ is $a$ distinguished triangle of $\mathrm{C}$, then $b \in \mathrm{A}$. 
(ii) $\left(\mathrm{A}, \mathrm{A}^{\perp}\right)$ is a torsion pair.

(iii) $A={ }^{\perp}\left(A^{\perp}\right)$.

(iv) $\mathfrak{A}=\operatorname{nc} n c \mathfrak{A}$.

Proof (i) $\Rightarrow$ (ii) holds by [11, Proposition 2.3(1)] since A is contravariantly finite because it has only finitely many indecomposable objects. (Indeed, C itself has only finitely many indecomposable objects.)

(ii) $\Rightarrow$ (iii) holds by the remarks following [11, Definition 2.2].

(iii) $\Rightarrow$ (i): If $\mathrm{X}$ is any full subcategory of $\mathrm{C}$ then ${ }^{\perp} \mathrm{X}$ is closed under extensions. Namely, if $a_{1}, a_{2} \in{ }^{\perp} \mathrm{X}$ and $a_{1} \rightarrow b \rightarrow a_{2} \rightarrow \Sigma a_{1}$ is a distinguished triangle, then each $x \in \mathrm{X}$ gives an exact sequence $\mathrm{C}\left(a_{2}, x\right) \rightarrow \mathrm{C}(b, x) \rightarrow \mathrm{C}\left(a_{1}, x\right)$. The outer terms are 0 , so $\mathrm{C}(b, x)=0$ whence $b \in{ }^{\perp} \mathrm{X}$.

(iii) $\Leftrightarrow$ (iv) follows from the remarks before the proposition by which $\mathrm{A}^{\perp}$ corresponds to $\Sigma$ nc $\mathfrak{A}$ and ${ }^{\perp}\left(\mathrm{A}^{\perp}\right)$ corresponds to $\Sigma^{-1} \operatorname{nc}(\Sigma \mathrm{nc} \mathfrak{A})=\operatorname{nc}$ nc $\mathfrak{A}$.

Remark 2.4 Note that by an easy argument, in a torsion pair $(\mathrm{X}, \mathrm{Y})$ we always have $\mathrm{Y}=\mathrm{X}^{\perp}$; see [11, Definition 2.2]. It follows that every torsion pair in $\mathrm{C}$ has the form $\left(A, A^{\perp}\right)$ for one of the subcategories $A$ in Proposition 2.3. By the proposition, there is hence a bijection between torsion pairs in $\mathrm{C}$ and sets of diagonals $\mathfrak{A}$ with $\mathfrak{A}=$ nc nc $\mathfrak{A}$.

Let $\mathcal{P}$ be the set of Ptolemy diagrams in polygons of any size with a distinguished base edge. For convenience, we will consider the edges of the polygon to be part of a Ptolemy diagram. Moreover, $\mathcal{P}$ includes the degenerate Ptolemy diagram consisting of two vertices and the distinguished base edge. We give a different (global) description of Ptolemy diagrams by establishing a recursive combinatorial equation for $\mathcal{P}$.

Recall that a polygon with no diagonals is called an empty cell and that a polygon with all diagonals is called a clique; these are both Ptolemy diagrams.

Proposition 2.5 The set $\mathcal{P}$ is recursively given as the disjoint union of

(i) The degenerate Ptolemy diagram,

(ii) An empty cell with at least three edges, one of which is the distinguished base edge, where we have glued onto each other edge an element of $\mathcal{P}$ along its distinguished base edge,

(iii) A clique with at least four edges, one of which is the distinguished base edge, where we have glued onto each other edge an element of $\mathcal{P}$ along its distinguished base edge.

These types correspond to the three parts of the right hand side of the equation in Fig. 4. In particular, a Ptolemy diagram can be decomposed completely into Ptolemy diagrams which are either empty cells or cliques.

Proof It is clear that the sets (i), (ii), and (iii) are disjoint.

Let a non-degenerate Ptolemy diagram $\mathfrak{A}$ be given with distinguished base edge $\{\alpha, \beta\}$. We will show that $\mathfrak{A}$ is either of type (ii) or type (iii). For convenience, we will 


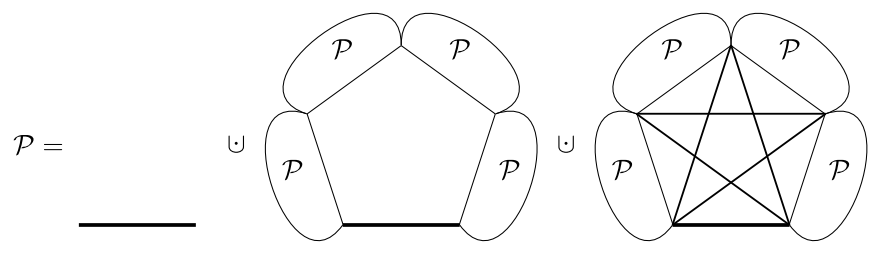

Fig. 4 The decomposition of the set of Ptolemy diagrams with a distinguished base edge

consider the vertices of the polygon to be ordered in an obvious way, starting with $\alpha$ and ending with $\beta$.

Type (ii): Suppose that there do not exist crossing diagonals $\mathfrak{a}$ and $\mathfrak{b}$ in $\mathfrak{A}$ ending in $\alpha$, respectively $\beta$. We will show that $\mathfrak{A}$ is of type (ii).

Consider increasing sequences of vertices $\alpha, \gamma_{1}, \ldots, \gamma_{m}, \beta$ with $m \geq 1$ for which the edges and diagonals

$$
\left\{\alpha, \gamma_{1}\right\},\left\{\gamma_{1}, \gamma_{2}\right\}, \ldots,\left\{\gamma_{m-1}, \gamma_{m}\right\},\left\{\gamma_{m}, \beta\right\}
$$

are in $\mathfrak{A}$, and choose a sequence with $m$ minimal. For ease of notation, write $\gamma_{0}=\alpha$ and $\gamma_{m+1}=\beta$. The displayed edges and diagonals along with the distinguished base edge $\{\alpha, \beta\}$ bound a region $C$.

We show that $\mathfrak{A}$ is of type (ii) by showing that no diagonal in $\mathfrak{A}$ intersects the interior of $C$. Then $C$ is an empty cell and each $\left\{\gamma_{j}, \gamma_{j+1}\right\}$ with $0 \leq j \leq m$ divides $C$ from a (smaller) Ptolemy diagram; see Fig. 4. Note that each smaller Ptolemy diagram is clearly uniquely determined.

Suppose that $\mathfrak{A}$ does contain a diagonal $\left\{\epsilon_{1}, \epsilon_{2}\right\}$ intersecting the interior of $C$. We can assume $\epsilon_{1}<\epsilon_{2}$. There are three cases, each leading to a contradiction.

(a) $\epsilon_{1}$ and $\epsilon_{2}$ are among the $\gamma_{i}$. Then $\epsilon_{1}=\gamma_{j-1}$ and $\epsilon_{2}=\gamma_{k+1}$ where $1 \leq j \leq k \leq m$. This contradicts that $m$ is minimal.

(b) One of $\epsilon_{1}$ and $\epsilon_{2}$ is among the $\gamma_{i}$ and the other is not, see Fig. 5. By symmetry, we can assume $\epsilon_{1}=\gamma_{j-1}$ and $\gamma_{k}<\epsilon_{2}<\gamma_{k+1}$ with $1 \leq j \leq k \leq m$. The diagonals $\left\{\epsilon_{1}, \epsilon_{2}\right\}=\left\{\gamma_{j-1}, \epsilon_{2}\right\}$ and $\left\{\gamma_{k}, \gamma_{k+1}\right\}$ cross. By the Ptolemy condition, $\mathfrak{c}=\left\{\gamma_{j-1}, \gamma_{k+1}\right\}$ is in $\mathfrak{A}$.

If $\mathfrak{c}$ intersects the interior of $C$ then we are in case (a). If it does not, then we must have $\gamma_{j-1}=\alpha$ and $\gamma_{k+1}=\beta$. But then there are crossing diagonals $\mathfrak{a}=\left\{\alpha, \epsilon_{2}\right\}=\left\{\gamma_{j-1}, \epsilon_{2}\right\}=\left\{\epsilon_{1}, \epsilon_{2}\right\}$ and $\mathfrak{b}=\left\{\beta, \gamma_{k}\right\}=\left\{\gamma_{k}, \gamma_{k+1}\right\}$ ending in $\alpha$, respectively $\beta$, contradicting our assumption on $\mathfrak{A}$.

(c) $\epsilon_{1}$ and $\epsilon_{2}$ are not among the $\gamma_{i}$, see Fig. 5. Then $\gamma_{j-1}<\epsilon_{1}<\gamma_{j}$ and $\gamma_{k}<\epsilon_{2}<$ $\gamma_{k+1}$ for some $1 \leq j \leq k \leq m$. The diagonal $\left\{\epsilon_{1}, \epsilon_{2}\right\}$ crosses each of the diagonals $\left\{\gamma_{j-1}, \gamma_{j}\right\}$ and $\left\{\gamma_{k}, \gamma_{k+1}\right\}$ so, by the Ptolemy condition, each of the diagonals $\left\{\epsilon_{1}, \gamma_{k+1}\right\}$ and $\left\{\gamma_{j-1}, \epsilon_{2}\right\}$ is in $\mathfrak{A}$. These diagonals cross, so by the Ptolemy condition $\mathfrak{c}=\left\{\gamma_{j-1}, \gamma_{k+1}\right\}$ is in $\mathfrak{A}$. Now conclude the argument by using the second paragraph of (b).

Type (iii): Suppose that crossing diagonals $\mathfrak{a}=\{\alpha, \delta\}$ and $\mathfrak{b}=\left\{\beta, \delta^{\prime}\right\}$ ending in $\alpha$, respectively $\beta$, do exist in $\mathfrak{A}$. We will show that $\mathfrak{A}$ is of type (iii). 


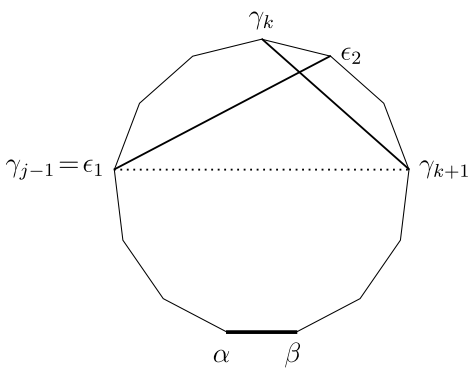

Type (ii), case (b)

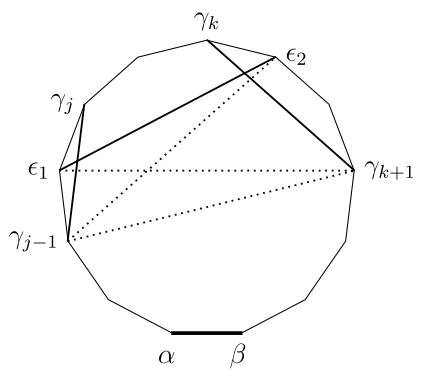

Type (ii), case (c)

Fig. 5 In type (ii), the diagonal $\left\{\epsilon_{1}, \epsilon_{2}\right\}$ forces the presence of the diagonal $\left\{\gamma_{j-1}, \gamma_{k+1}\right\}$

By the Ptolemy condition, $\left\{\alpha, \delta^{\prime}\right\}$ and $\{\beta, \delta\}$ are in $\mathfrak{A}$. Consider those vertices which are connected to each of $\alpha$ and $\beta$ by an edge or a diagonal in $\mathfrak{A}$. Denote them by $\delta_{1}, \ldots, \delta_{m}$ in increasing order and note that $m \geq 2$ because $\delta$ and $\delta^{\prime}$ are among the $\delta_{i}$. For ease of notation write $\delta_{0}=\alpha$ and $\delta_{m+1}=\beta$.

Let $0 \leq j<k \leq m+1$. Then $\left\{\delta_{j}, \delta_{k}\right\}$ is in $\mathfrak{A}$. Namely, this holds by definition if $j=0$ since then $\delta_{j}=\alpha$. So we can assume $1 \leq j$ and by symmetry $k \leq m$. But then $\left\{\alpha, \delta_{k}\right\}$ and $\left\{\delta_{j}, \beta\right\}$ are crossing diagonals in $\mathfrak{A}$ and by the Ptolemy condition $\left\{\delta_{j}, \delta_{k}\right\}$ is in $\mathfrak{A}$. So the $\delta_{i}$ form the vertices of a clique of edges and diagonals in $\mathfrak{A}$ which contains the distinguished base edge.

We show that $\mathfrak{A}$ is of type (iii) by showing that if $\left\{\delta_{j}, \delta_{j+1}\right\}$ is a diagonal with $0 \leq j \leq m$, then no diagonal in $\mathfrak{A}$ crosses $\left\{\delta_{j}, \delta_{j+1}\right\}$ : then $\left\{\delta_{j}, \delta_{j+1}\right\}$ divides the clique with vertices $\delta_{i}$ from a (smaller) Ptolemy diagram; see Fig. 4. Note that, again, each smaller Ptolemy diagram is uniquely determined.

So suppose that $\mathfrak{A}$ contains a diagonal $\left\{\epsilon_{1}, \epsilon_{2}\right\}$ crossing $\left\{\delta_{j}, \delta_{j+1}\right\}$. We can assume that $\epsilon_{1}<\epsilon_{2}$ and by symmetry considerations that $\delta_{j}<\epsilon_{1}<\delta_{j+1}$. Note that this entails $j \leq m-1$.

There are two cases, each leading to a contradiction.

(a) $\epsilon_{2} \neq \beta$, see Fig. 6 . Then the diagonal $\left\{\epsilon_{1}, \epsilon_{2}\right\}$ crosses the diagonals $\left\{\alpha, \delta_{j+1}\right\}$ and $\left\{\beta, \delta_{j+1}\right\}$ so, by the Ptolemy condition, $\left\{\alpha, \epsilon_{1}\right\}$ and $\left\{\beta, \epsilon_{1}\right\}$ are in $\mathfrak{A}$. Hence $\epsilon_{1}$ is among the $\delta_{i}$, contradicting $\delta_{j}<\epsilon_{1}<\delta_{j+1}$.

(b) $\epsilon_{2}=\beta$, see Fig. 6. Then $\left\{\beta, \epsilon_{1}\right\}=\left\{\epsilon_{1}, \epsilon_{2}\right\}$ is in $\mathfrak{A}$. Moreover, $\left\{\epsilon_{1}, \epsilon_{2}\right\}$ crosses $\left\{\alpha, \delta_{j+1}\right\}$ so, by the Ptolemy condition, $\left\{\alpha, \epsilon_{1}\right\}$ is in $\mathfrak{A}$. Hence $\epsilon_{1}$ is again among the $\delta_{i}$, which is a contradiction.

Remark 2.6 The proposition proves Theorem A(ii) from the introduction: each Ptolemy diagram can be uniquely decomposed into regions, each of which is either an empty cell or a clique.

Moreover, let $\mathfrak{A}$ be a Ptolemy diagram. To obtain nc $\mathfrak{A}$ from $\mathfrak{A}$, one replaces empty cells by cliques and vice versa in the decomposition.

Namely, let $\mathfrak{d}$ be an arbitrary diagonal. If $\mathfrak{d}$ separates two regions of $\mathfrak{A}$, then $\mathfrak{d}$ is one of the diagonals along which two smaller Ptolemy diagrams have been glued in 
Fig. 6 In type (iii), the diagonal $\left\{\epsilon_{1}, \epsilon_{2}\right\}$ forces the presence of the diagonals $\left\{\alpha, \epsilon_{1}\right\}$ and $\left\{\beta, \epsilon_{1}\right\}$

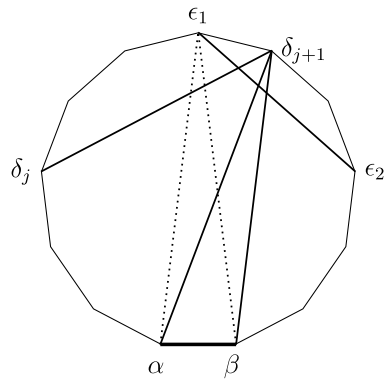

Type (iii), case (a)

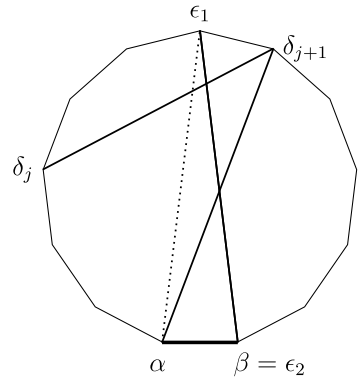

Type (iii), case (b)

the decomposition to form $\mathfrak{A}$ so, clearly, $\mathfrak{d}$ crosses no diagonal of $\mathfrak{A}$, thus $\mathfrak{d} \in$ nc $\mathfrak{A}$. If $\mathfrak{d}$ is an internal diagonal in a clique, then it crosses some other internal diagonal which must be in $\mathfrak{A}$, so $\mathfrak{d} \notin$ nc $\mathfrak{A}$. If $\mathfrak{d}$ is an internal diagonal in an empty cell, then it crosses no diagonal of $\mathfrak{A}$, so $\mathfrak{d} \in$ nc $\mathfrak{A}$.

Note that we have $\mathfrak{A}=\mathrm{nc} \mathfrak{A}$ if and only if $\mathfrak{A}$ is a triangulation of the polygon, since a triangle is the only polygon which is an empty cell and a clique simultaneously.

With the above decomposition, we can show the following alternative characterization of Ptolemy diagrams.

Proposition 2.7 We have $\mathfrak{A}=\mathrm{nc} n \mathrm{~A} \mathfrak{A}$ if and only if $\mathfrak{A}$ is a Ptolemy diagram.

Proof Suppose that $\mathfrak{A}=$ nc nc $\mathfrak{A}$. In Fig. 1 , consider the diagonal $\left\{\alpha_{1}, \beta_{1}\right\}$. The diagonals crossing it are precisely the diagonals which connect a vertex on one side of $\left\{\alpha_{1}, \beta_{1}\right\}$ with a vertex on the other side of $\left\{\alpha_{1}, \beta_{1}\right\}$. But each such diagonal intersects $\mathfrak{a}$ or $\mathfrak{b}$ so is outside nc $\mathfrak{A}$. Hence $\left\{\alpha_{1}, \beta_{1}\right\}$ is in nc nc $\mathfrak{A}=\mathfrak{A}$. The other diagonals in the Ptolemy condition follow similarly.

Conversely, suppose that $\mathfrak{A}$ satisfies the Ptolemy condition. By Remark 2.6, the operator nc interchanges empty cells and cliques in the decomposition of $\mathfrak{A}$ according to Proposition 2.5, so it is clear that $\mathfrak{A}=$ nc nc $\mathfrak{A}$.

Remark 2.8 Combining Remark 2.4 and Proposition 2.7 proves Theorem A(i) of the introduction. In particular, to count torsion pairs in the cluster category of type $A_{n}$ we only need to determine the number of Ptolemy diagrams of the $(n+3)$-gon with a distinguished base edge.

\section{Counting the number of Ptolemy diagrams}

In this section, we deduce expressions for the number of Ptolemy diagrams. First, we compute the number of Ptolemy diagrams with a distinguished base edge. In a second step, we also determine the number of Ptolemy diagrams up to rotation. 


\subsection{Ptolemy diagrams with a distinguished base edge}

Using combinatorial reasoning, we shall obtain below an equation for the (ordinary) generating function

$$
\mathcal{P}(y)=\sum_{N \geq 1} \#\{\text { Ptolemy diagrams of the }(N+1) \text {-gon }\} y^{N} .
$$

Let us briefly recall some facts from the general theory of generating functions, see, for example, the book by Bergeron, Labelle and Leroux [4, Sect. 1.3] or Aigner [1, Sects. 3.2 and 3.3]. Of course, our objective is to convey the general idea, precise formulations are given in the cited textbooks.

Let $\mathcal{F}$ and $\mathcal{G}$ be sets of objects. Each object is assigned to a non-negative integer, referred to as its size. Let $\mathcal{F}(y)$ and $\mathcal{G}(y)$ be their generating functions. Then the generating function

- For the disjoint union of $\mathcal{F}$ and $\mathcal{G}$ is $\mathcal{F}(y)+\mathcal{G}(y)$, and

- For the set of objects obtained by pairing objects from $\mathcal{F}$ and $\mathcal{G}$ is $\mathcal{F}(y) \mathcal{G}(y)$, where the size of a pair is the sum of the sizes of its two components.

Because of the natural correspondence with the operation on generating functions, we denote the pairing of sets considered in the second item by $\mathcal{F} \cdot \mathcal{G}$.

We can now derive an equation for the generating function of lists of Ptolemy $\operatorname{diagrams} \mathcal{L}^{\mathcal{P}}$. Namely, either such a list is empty, or it is a pair whose first component is a Ptolemy diagram and whose second component is a list of Ptolemy diagrams. We thus have

$$
\mathcal{L}^{\mathcal{P}}=\emptyset \bullet \mathcal{P} \cdot \mathcal{L}^{\mathcal{P}}
$$

or, on the level of generating functions,

$$
\mathcal{L}^{\mathcal{P}}(y)=1+\mathcal{P}(y) \mathcal{L}^{\mathcal{P}}(y),
$$

which entails

$$
\mathcal{L}^{\mathcal{P}}(y)=\frac{1}{1-\mathcal{P}(y)} .
$$

Clearly, we can interpret the set of Ptolemy diagrams of type (ii) in Proposition 2.5 as the set of lists of Ptolemy diagrams with at least two elements. With a slight shift of perspective, this is the same as a triple whose first two components are Ptolemy diagrams and whose last component is a list of diagrams. Hence, this set has generating function $\mathcal{P}(y)^{2} /(1-\mathcal{P}(y))$. Similarly, a Ptolemy diagram of type (iii) in Proposition 2.5 can be interpreted as a list of diagrams with at least three elements. Namely, recall that in the decomposition of Proposition 2.5, the cliques which occur have at least four edges, one of which is the distinguished base edge; to the other three, we can attach Ptolemy diagrams.

In summary, using the combinatorial decomposition of Proposition 2.5 sketched in Fig. 4,

$$
\mathcal{P}(y)=y+\frac{\mathcal{P}(y)^{2}}{1-\mathcal{P}(y)}+\frac{\mathcal{P}(y)^{3}}{1-\mathcal{P}(y)} .
$$


Let us rewrite this equation (essentially multiplying by $1-\mathcal{P}(y)$ ), to make it amenable to Lagrange inversion (e.g. [4, Sect. 3.1] or [1, Theorem 3.8]):

$$
\mathcal{P}(y)=y \frac{1-\mathcal{P}(y)}{1-2 \mathcal{P}(y)-\mathcal{P}(y)^{2}},
$$

i.e. $\mathcal{P}(y)=y A(\mathcal{P}(y))$ with $A(y)=(1-y) /\left(1-2 y-y^{2}\right)$. Thus, denoting the coefficient of $y^{N}$ in $\mathcal{P}(y)$ with $\left[y^{N}\right] \mathcal{P}(y)$, we have

$$
\left[y^{N}\right] \mathcal{P}(y)=\frac{1}{N}\left[y^{N-1}\right]\left(\frac{1-y}{1-2 y-y^{2}}\right)^{N} .
$$

We can now apply the binomial theorem $(1+z)^{a}=\sum_{k>0}\left(\begin{array}{l}a \\ k\end{array}\right) z^{k}$, for $a \in \mathbb{Z}$ and $\left(\begin{array}{l}a \\ k\end{array}\right)=$ $a(a-1) \cdots(a-k+1) / k$ !, to transform the right hand side into a sum. As pointed out by Christian Krattenthaler the result becomes much nicer if we first rewrite the expression slightly, taking advantage of the fact that $1-2 y-y^{2}$ is 'almost' $(1-y)^{2}$ :

$$
\begin{aligned}
(1-y)^{N}\left(1-2 y-y^{2}\right)^{-N} & =(1-y)^{-N}\left(1-\frac{2 y^{2}}{(1-y)^{2}}\right)^{-N} \\
& =(1-y)^{-N} \sum_{\ell \geq 0}\left(\begin{array}{c}
-N \\
\ell
\end{array}\right)(-1)^{\ell} \frac{\left(2 y^{2}\right)^{\ell}}{(1-y)^{2 \ell}} \\
& =\sum_{\ell \geq 0}\left(\begin{array}{c}
-N \\
\ell
\end{array}\right)(-1)^{\ell}\left(2 y^{2}\right)^{\ell} \sum_{k \geq 0}\left(\begin{array}{c}
-N-2 \ell \\
k
\end{array}\right)(-1)^{k} y^{k} \\
& =\sum_{k, \ell \geq 0}\left(\begin{array}{c}
-N \\
\ell
\end{array}\right)\left(\begin{array}{c}
-N-2 \ell \\
k
\end{array}\right)(-1)^{k+\ell} 2^{\ell} y^{k+2 \ell} .
\end{aligned}
$$

Extracting the coefficient of $y^{N-1}$ in (3) by setting $k=N-1-2 \ell$, we obtain

$$
\left[y^{N}\right] \mathcal{P}(y)=\frac{1}{N} \sum_{\ell \geq 0}\left(\begin{array}{c}
-N \\
\ell
\end{array}\right)\left(\begin{array}{c}
-N-2 \ell \\
N-1-2 \ell
\end{array}\right)(-1)^{N-1-\ell} 2^{\ell} .
$$

Finally, using $\left(\begin{array}{c}-N \\ \ell\end{array}\right)=(-1)^{\ell}\left(\begin{array}{c}N+\ell-1 \\ \ell\end{array}\right)$, we get that the number of Ptolemy diagrams of an $(N+1)$-gon with a distinguished base edge is

$$
\frac{1}{N} \sum_{\ell \geq 0} 2^{\ell}\left(\begin{array}{c}
N-1+\ell \\
\ell
\end{array}\right)\left(\begin{array}{c}
2 N-2 \\
N-1-2 \ell
\end{array}\right)
$$

Setting $N=n+2$ proves Theorem B of the introduction, and the first few values are given there.

Remark 3.1 Note that Petkovšek's algorithm hyper [17, Sect. 8] proves that the sum above cannot be written as a linear combination of (a fixed number of) hypergeometric terms. 
Remark 3.2 Since the generating function $\mathcal{P}(y)$ satisfies an algebraic equation, the asymptotic behaviour of the coefficients of $\mathcal{P}(y)$ can be extracted automatically, for example, using the equivalent function in Bruno Salvy's package gdev available at http://algo.inria.fr/libraries/. Thus, we learn that the leading term of the asymptotic expansion of $\left[y^{N}\right] \mathcal{P}(y)$ is

$$
\frac{\alpha}{\sqrt{\pi N^{3}}} \rho^{N}
$$

where $\rho=6.847333996370022 \ldots$ is the largest positive root of $8 x^{3}-48 x^{2}-47 x+4$ and $\alpha=0.10070579427884086 \ldots$ is the smallest positive root of $1136 x^{6}-71 x^{4}-$ $98 x^{2}+1$.

\subsection{Ptolemy diagrams up to rotation}

Let us now turn to the enumeration of Ptolemy diagrams up to rotation. It seems easiest to apply a relatively general technique known as the 'dissymmetry theorem for trees'. Namely, we will consider Ptolemy diagrams as certain planar trees, where each inner vertex of the tree corresponds to either an empty cell or a clique of the diagram. Thus, we will have to count trees according to their number of leaves, where the edges incident to an inner vertex are cyclically ordered and additionally these inner vertices 'know' whether they correspond to an empty cell or a clique. This situation is covered by Proposition 3.3 below.

This proposition is phrased in the language of combinatorial species (as described in [4]), which is at first a tool to compute with labelled objects. Formally, a species is a functor from the category of finite sets with bijections into itself. Thus, applying a species $\mathcal{F}$ to a finite set $U$, namely a set of labels, we obtain a new set $\mathcal{F}[U]$, namely the set of objects that can be produced using the given labels. Applying $\mathcal{F}$ to a bijection $\sigma: U \rightarrow V$ produces a bijection $\mathcal{F}[\sigma]: \mathcal{F}[U] \rightarrow \mathcal{F}[V]$, which, by functoriality, corresponds to relabelling the objects. (However, when defining a particular species here, we refrain from giving a precise definition of this relabelling operation.)

A simple, but nevertheless important species is the singleton species $Y$ : it returns the input set $U$ if $U$ has cardinality one and otherwise the empty set. Another basic species we will need is the species of unordered pairs $E_{2}$, which returns the input set $U$ if $U$ has cardinality two and the empty set otherwise. Finally, for $k \geq 1$ we introduce the species of cycles $C_{k}$, which consists of all (oriented) cycles with $k$ labelled vertices.

We associate to every species $\mathcal{F}$ a so-called exponential generating function $\mathcal{F}(y)$, which is given by

$$
\mathcal{F}(y)=\sum_{N \geq 1} \# \mathcal{F}[\{1,2, \ldots, N\}] \frac{y^{N}}{N !},
$$

i.e. the coefficient of $y^{N}$ is the number of objects with labels $\{1,2, \ldots, N\}$ produced by $\mathcal{F}$, divided by $N$ !. In particular, the exponential generating function associated to $Y$ is $Y(y)=y$, and the exponential generating function associated to $E_{2}$ is $E_{2}(y)=$ $y^{2} / 2$. Finally, $C_{k}(y)=(k-1) ! \frac{y^{k}}{k !}=\frac{y^{k}}{k}$. 
There are natural definitions for the sum $\mathcal{F}+\mathcal{G}$, the product $\mathcal{F} \cdot \mathcal{G}$ and the composition $\mathcal{F} \circ \mathcal{G}$ of two species $\mathcal{F}$ and $\mathcal{G}$. We only give informal descriptions of the sets of objects which they produce, and refer for precise definitions to [4, Sect. 1]. Let $U$ be a set of labels, then

- The set of objects in $(\mathcal{F}+\mathcal{G})[U]$ is the disjoint union of $\mathcal{F}[U]$ and $\mathcal{G}[U]$;

- The set of objects in $(\mathcal{F} \cdot \mathcal{G})[U]$ is obtained by partitioning the set $U$ in all possible ways into two disjoint (possibly empty) sets $V$ and $W$ such that $U=V \cup W$, and producing all pairs of objects in

$$
(\mathcal{F}[V], \mathcal{G}[W]),
$$

i.e. $\{(f, g) \mid f \in \mathcal{F}[V], g \in \mathcal{G}[W]\}$;

- The set of objects in $(\mathcal{F} \circ \mathcal{G})[U]$ is the set of all tuples of the form

$$
\left(\mathcal{F}[\{1,2, \ldots, k\}], \mathcal{G}\left[B_{1}\right], \mathcal{G}\left[B_{2}\right], \ldots, \mathcal{G}\left[B_{k}\right]\right),
$$

where $\left\{B_{1}, B_{2}, \ldots, B_{k}\right\}$ is a set partition of $U$.

The composition of species can be visualised by taking an object produced by $\mathcal{F}$, and replacing all its labels by objects produced by $\mathcal{G}$, such that the set of labels is exactly $U$. In particular, $\mathcal{F} \circ Y=Y \circ \mathcal{F}=\mathcal{F}$.

Finally, we need to describe the derivative $\mathcal{F}^{\prime}$ of a species $\mathcal{F}$. Given a set of labels $U$, we set $\mathcal{F}^{\prime}[U]=\mathcal{F}[U \cup\{*\}]$, where $*$ is a 'transcendental' element, i.e. an element that does not appear in $U$.

It should not come as a surprise (although it certainly needs a proof) that the exponential generating functions associated to the sum, the product, the composition, and the derivative of species are respectively $\mathcal{F}(y)+\mathcal{G}(y), \mathcal{F}(y) \cdot \mathcal{G}(y), \mathcal{F}(\mathcal{G}(y))$ and $\mathcal{F}^{\prime}(y)$.

It remains to introduce the species of $R$-enriched trees $b_{R}$ and $R^{\prime}$-enriched rooted trees $B_{R^{\prime}}$ with labels on the leaves, see [4, Definition 13, Sect. 3.1 and p. 287, Sect. 4.1]: let $R$ be a species with $\# R[\emptyset]=0, \# R[\{1\}]=1$ and $\# R[\{1,2\}]=0$. Then an $R$-enriched tree on a set of labels $U$ is a tree with at least two vertices, whose vertices of degree one (i.e. the leaves) correspond to the labels in $U$. Additionally, every vertex is assigned an object from $R[N]$, where $N$ is the set of neighbours of the vertex. Since $\# R[\{1,2\}]=0$, there are no vertices of degree two. Therefore, any such tree must have more leaves than inner vertices and thus the set of $R$-enriched trees with a finite number of leaves is finite. The condition $\# R[\{1\}]=1$ implies that only the inner vertices carry additional structure.

An $R^{\prime}$-enriched rooted tree on a set of labels $U$ is a rooted tree, possibly an isolated vertex, where the vertices of degree at most one (i.e. the leaves) correspond to the labels in $U$. Additionally, every vertex is assigned an object from $R^{\prime}[N]$, where $N$ is the set of those neighbours of the vertex which are further away from the root than the vertex itself. Again, since $\# R^{\prime}[\{1\}]=0$, no vertex can have a single successor, and thus the set of $R^{\prime}$-enriched rooted trees with a finite number of leaves is finite.

In our situation, we set $R=Y+C_{\geq 3}+C_{\geq 4}$ where $C_{\geq k}$ denotes the species of cycles with at least $k$ vertices. The derivative of $R$ is

$$
R^{\prime}=1+\mathcal{L}_{\geq 2}+\mathcal{L}_{\geq 3},
$$


Fig. 7 The correspondence between $R^{\prime}$-enriched rooted trees and labelled Ptolemy diagrams with base edge

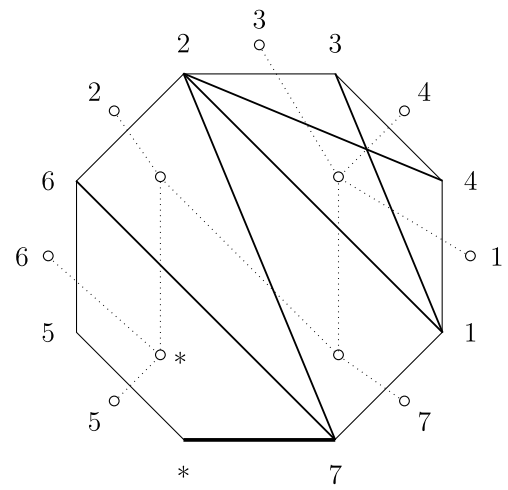

where $\mathcal{L}_{\geq k}$ denotes the species of lists with at least $k$ elements. We now see that $B_{R^{\prime}}$ is isomorphic (in the sense of [4, Definition 12, Sect. 1.2]) to the combinatorial species of Ptolemy diagrams with a distinguished base edge and labels on all vertices except the counterclockwise first on the base edge. Namely, a Ptolemy diagram can be regarded as an $R^{\prime}$-enriched rooted tree as follows: the region attached to the distinguished base edge corresponds to the root and the other regions to the internal vertices of the tree, i.e. vertices which are not leaves, see Fig. 7. Note that the degenerate Ptolemy diagram, consisting of the base edge only, carries one label. This corresponds to the tree consisting of one isolated vertex, which is also labelleddespite being the root of the tree.

Let us informally explain the meaning of the three summands in $R^{\prime}$ : the first summand, 1, applies if a vertex is a leaf and thus has no successor. The second summand, $\mathcal{L}_{\geq 2}$, applies if a vertex corresponds to a region that is of type (ii) in the decomposition of Proposition 2.5, i.e. an empty cell, in which case the vertex must have at least two successors. Finally, the third summand, $\mathcal{L}_{\geq 3}$, applies if a vertex corresponds to a region that is of type (iii) in Proposition 2.5, in which case the vertex must have at least three successors. In the latter two cases, the species of lists imposes an ordering onto the successors of the vertex.

In a similar manner, we can see that $b_{R}$ is the species of Ptolemy diagrams up to rotation and labels on all vertices. Here, enriching the inner vertices with the species of cycles imposes a cyclic ordering on the neighbours of each vertex.

We can now state the announced tool. We reproduce it here in a slightly simplified form; it is the special case of Theorem 4.1.7 in [4] obtained by setting $X=1$. In this special case, we additionally have to require $\# R_{0}[\{1,2\}]=0$ to ensure welldefinedness of the species involved.

Proposition 3.3 Let $R_{0}$ be a combinatorial species such that $\# R_{0}[\emptyset]=\# R_{0}[\{1\}]=$ $\# R_{0}[\{1,2\}]=0$ and let $R=R_{0}+Y$. Then the combinatorial species $b_{R}$ of $R$ enriched trees and the combinatorial species of $R^{\prime}$-enriched rooted trees $B_{R^{\prime}}$ are related as follows:

$$
b_{R}+B_{R^{\prime}}^{2}=\left(E_{2}+R_{0}\right) \circ B_{R^{\prime}}+Y \cdot B_{R^{\prime}} .
$$


As far as the enumeration of labelled structures is concerned, this proposition is not very interesting. Namely, it follows directly from the definition of the derivative of a species that $B_{R^{\prime}}$ is the derivative of $b_{R}$ : the correspondence is accomplished by making the root into another labelled vertex. In particular, the number of labelled Ptolemy diagrams up to rotation with $N+1$ vertices (and $N+1$ labels) equals the number of labelled Ptolemy diagrams with distinguished base edge with $N+1$ vertices (and $N$ labels) and is given by $N$ ! times the $N$ th coefficient of $\mathcal{P}(y)$.

However, the proposition enables us to determine also the (ordinary) generating function of unlabelled Ptolemy diagrams up to rotation. In the jargon of combinatorial species, this is the isomorphism type generating function $\widetilde{b}_{R}(y)$ of the species $b_{R}$ with the specific value of $R$ used above. In general, the isomorphism type generating function of a species $\mathcal{F}$ is denoted $\widetilde{\mathcal{F}}(y)$ and we have the usual rules $(\widetilde{\mathcal{F}+\mathcal{G}})(y)=$ $\widetilde{\mathcal{F}}(y)+\widetilde{\mathcal{G}}(y)$ and $\widetilde{(\mathcal{F G})}(y)=\widetilde{\mathcal{F}}(y) \widetilde{\mathcal{G}}(y)$. To compute $\tilde{b}_{R}(y)$, we additionally need to use cycle indicator series. We collect the facts significant for us in the following lemma.

Lemma 3.4 Let $\mathcal{F}$ be a combinatorial species and $Z_{\mathcal{F}}$ its cycle indicator series. Then the generating function for the isomorphism types of $\mathcal{F}$ is given by

$$
\widetilde{\mathcal{F}}=Z_{\mathcal{F}}\left(y, y^{2}, y^{3}, \ldots\right) \quad(\text { see }[4, \text { Theorem } 8, \text { Sect. 1.2] }) .
$$

Moreover, let $\mathcal{G}$ be another species satisfying $\# \mathcal{G}[\emptyset]=0$. Then the generating function for the isomorphism types of $\mathcal{F} \circ \mathcal{G}$ is given by

$$
\widetilde{\mathcal{F} \circ \mathcal{G}}=Z_{\mathcal{F}}\left(\widetilde{\mathcal{G}}(y), \widetilde{\mathcal{G}}\left(y^{2}\right), \widetilde{\mathcal{G}}\left(y^{3}\right), \ldots\right) \quad(\text { see }[4, \text { Theorem } 2, \text { Sect. 1.4] })
$$

The cycle indicator series of the species of cycles $C$ is given by

$$
Z_{C}\left(p_{1}, p_{2}, \ldots\right)=\sum_{d \geq 1} \frac{\phi(d)}{d} \log \left(\frac{1}{1-p_{d}}\right)
$$

where $\phi$ is Euler's totient (see [4, (18), Sect. 1.4]).

The cycle indicator series of the two element set $E_{2}$ (which coincides with the 2-cycle $C_{2}$ ) is given by

$$
Z_{E_{2}}\left(p_{1}, p_{2}, \ldots\right)=\frac{1}{2}\left(p_{1}^{2}+p_{2}\right) \quad(\text { see }[4, \text { Table } 5, \text { App. } 2])
$$

The cycle indicator series of the 3-cycle $C_{3}$ is given by

$$
Z_{C_{3}}\left(p_{1}, p_{2}, \ldots\right)=\frac{1}{3}\left(p_{1}^{3}+2 p_{3}\right) \quad(\text { see }[4, \text { Table } 5, \text { App. 2] }) .
$$

Note that, since $\mathcal{P}(y)$ is algebraic, $\widetilde{\mathcal{P}}(y)=\mathcal{P}(y)$. Putting all the bits together, we find: 
Proposition 3.5 The generating function for Ptolemy diagrams up to rotation is

$$
\begin{aligned}
& 2 \sum_{d \geq 1} \frac{\phi(d)}{d} \log \left(\frac{1}{1-\mathcal{P}\left(y^{d}\right)}\right)-\frac{1}{2}\left(3 \mathcal{P}(y)^{2}+\mathcal{P}\left(y^{2}\right)\right)-\frac{1}{3}\left(\mathcal{P}(y)^{3}+2 \mathcal{P}\left(y^{3}\right)\right) \\
& \quad-2 \mathcal{P}(y)+y \mathcal{P}(y),
\end{aligned}
$$

where $\mathcal{P}(y)$ is the generating function for Ptolemy diagrams with a distinguished base edge, and $\phi(d)$ is Euler's totient.

The first few coefficients are given in the introduction.

Proof We use Proposition 3.3 with $R_{0}=C_{\geq 3}+C_{\geq 4}$. Since (formally) $E_{2}+R_{0}=$ $C_{k \geq 2}+C_{k \geq 4}=2 C-2 Y-E_{2}-C_{3}$,

$$
Z_{E_{2}+R_{0}}=2 \sum_{d \geq 1} \frac{\phi(d)}{d} \log \left(\frac{1}{1-p_{d}}\right)-2 p_{1}-\frac{1}{2}\left(p_{1}^{2}+p_{2}\right)-\frac{1}{3}\left(p_{1}^{3}+2 p_{3}\right) .
$$

Since $\mathcal{P}(y)$ is algebraic, we have $\widetilde{B_{R^{\prime}}}=\mathcal{P}(y)$ and therefore

$$
\begin{aligned}
\tilde{b}_{R}(y)= & Z_{E_{2}+R_{0}}\left(\mathcal{P}(y), \mathcal{P}\left(y^{2}\right), \ldots\right)+y \mathcal{P}(y)-\mathcal{P}(y)^{2} \\
= & 2 \sum_{d \geq 1} \frac{\phi(d)}{d} \log \left(\frac{1}{1-\mathcal{P}\left(y^{d}\right)}\right)-2 \mathcal{P}(y)-\frac{1}{2}\left(\mathcal{P}(y)^{2}+\mathcal{P}\left(y^{2}\right)\right) \\
& -\frac{1}{3}\left(\mathcal{P}(y)^{3}+2 \mathcal{P}\left(y^{3}\right)\right)+y \mathcal{P}(y)-\mathcal{P}(y)^{2},
\end{aligned}
$$

which is equivalent to the claim.

Acknowledgements We are grateful to Christian Krattenthaler for a very useful suggestion leading to the present formula in Theorem B which greatly improves a previous version. We also thank an anonymous referee for reading the paper very carefully, correcting an error, and suggesting several improvements to the presentation. The diagrams were typeset with TikZ.

\section{References}

1. Aigner, M.: A Course in Enumeration. Grad. Texts in Math., vol. 238. Springer, Berlin (2007)

2. Beilinson, A.A., Bernstein, J., Deligne, P.: Faisceaux pervers. Astérisque 100 (1982). (Vol. 1 of the proceedings of the conference "Analysis and topology on singular spaces", Luminy, 1981)

3. Beligiannis, A., Reiten, I.: Homological and homotopical aspects of torsion theories. Mem. Amer. Math. Soc. 188(883) (2007)

4. Bergeron, F., Labelle, G., Leroux, P.: Combinatorial Species and Tree-Like Structures. Encyclopedia Math. Appl., vol. 67. Cambridge University Press, Cambridge (1998)

5. Bondarko, M.V.: Weight structures vs. $t$-structures; weight filtrations, spectral sequences, and complexes (for motives and in general). K-Theory 6, 387-504 (2010)

6. Buan, A.B., Marsh, R.J., Reineke, M., Reiten, I., Todorov, G.: Tilting theory and cluster combinatorics. Adv. Math. 204, 572-618 (2006)

7. Caldero, P., Chapoton, F., Schiffler, R.: Quivers with relations arising from clusters $\left(A_{n}\right.$ case). Trans. Am. Math. Soc. 358, 1347-1364 (2006) 
8. Dickson, S.E.: A torsion theory for abelian categories. Trans. Am. Math. Soc. 121, 223-235 (1966)

9. Holm, T., Jørgensen, P.: On a cluster category of infinite Dynkin type, and the relation to triangulations of the infinity-gon. Math. Z. (2011, to appear). doi:10.1007/s00209-010-0797-z, 0902.4125v1 [math.RT]

10. Iyama, O.: Higher dimensional Auslander-Reiten theory on maximal orthogonal subcategories. Adv. Math. 210, 22-50 (2007)

11. Iyama, O., Yoshino, Y.: Mutation in triangulated categories and rigid Cohen-Macaulay modules. Invent. Math. 172, 117-168 (2008)

12. Keller, B.: On triangulated orbit categories. Doc. Math. 10, 551-581 (2005)

13. Keller, B., Reiten, I.: Cluster tilted algebras are Gorenstein and stably Calabi-Yau. Adv. Math. 211, 123-151 (2007)

14. Köhler, C.: Thick subcategories of finite algebraic triangulated categories. 1010.0146v1 [math.CT] (2010)

15. $\mathrm{Ng}, \mathrm{P} .:$ A characterization of torsion theories in the cluster category of Dynkin type $A_{\infty} .1005 .4364 \mathrm{v} 1$ [math.RT] (2010)

16. Pauksztello, D.: Compact corigid objects in triangulated categories and co- $t$-structures. Cent. Eur. J. Math. 6, 25-42 (2008)

17. Petkovšek, M., Wilf, H., Zeilberger, D.: $A=B$. AK Peters, Wellesley (1996)

18. Sloane, N.: The on-line encyclopedia of integer sequences. Published electronically at http://oeis.org (2010) 